\title{
Dornase Alfa Inhalation Solution
}

National Cancer Institute

\section{Source}

National Cancer Institute. Dornase Alfa Inhalation Solution. NCI Thesaurus. Code C70980.

A highly purified solution of recombinant human deoxyribonuclease I (rhDNAse) with selective DNA cleaving activity. Administrated through inhalation of the nebulized solution, dornase alpha catalyzes DNA degradation in viscous airway secretions, which may render airway secretions less viscous, thus promoting the clearing of airway mucous plugging and improvement in pulmonary function. 